\title{
Systems-level perspective of sudden infant death syndrome
}

\author{
Nathan Salomonis ${ }^{1}$
}

Sudden infant death syndrome (SIDS) remains one of the primary causes of infant mortality in developed countries. Although the causes of SIDS remain largely inconclusive, some of the most informative associations implicate molecular, genetic, anatomical, physiological, and environmental (i.e., infant sleep) factors. Thus, a comprehensive and evolving systems-level model is required to understand SIDS susceptibility. Such models, by being powerful enough to uncover indirect associations, could be used to expand our list of candidate targets for in-depth analysis. We present an integrated WikiPathways model for SIDS susceptibility that includes associated cell systems, signaling pathways, genetics, and animal phenotypes. Experimental and literature-based gene-regulatory data have been integrated into this model to identify intersecting upstream control elements and associated interactions. To expand this pathway model, we performed a comprehensive analysis of existing proteomics data from brainstem samples of infants with SIDS. From this analysis, we discovered changes in the expression of several proteins linked to known SIDS pathologies, including factors involved in glial cell production, hypoxia regulation, and synaptic vesicle release, in addition to interactions with annotated SIDS markers. Our results highlight new targets for further consideration that further enrich this pathway model, which, over time, can improve as a wiki-based, community curation project.

$S_{s}$ udden infant death syndrome (SIDS) is an idiopathic syndrome that results in the sudden and unexpected death of an infant (less than 1 year of age), almost always during deep sleep. It is diagnosed when no cause of death can be found by autopsy. A number of common biological risk factors have been linked to SIDS susceptibility, including age of the infant, sex, ethnicity, gestational prematurity, and prenatal maternal exposure to tobacco or alcohol (1). In addition, a number of environmental factors have been demonstrated to increase the likelihood of SIDS, including a prone sleeping position, the sleeping environment, infection, and stress (2). However, the lack of consistent diagnosis, difficulty in distinguishing SIDS from asphyxia, suffocation or shock, and (in most cases) the presence of multiple external risk factors make it challenging to identify the underlying cause of death.
Although no clear diagnostic markers currently exist, several polymorphisms have been identified that are significantly over-represented in distinct SIDS ethnic populations (3-14). The large majority of these polymorphisms exist in genes associated with neuronal signaling, cardiac contraction, and inflammatory responses. This diversity of potential innate risk factors, associated signaling pathways, and genetics makes it difficult to obtain an integrated view of SIDS susceptibility. Indeed, SIDS may be the result of multiple distinct disorders with a common endpoint-spontaneous death during sleeprather than a single disease entity. Although a large number of studies have been performed, there are several significant limitations for research in the area of SIDS molecular pathology. These limitations include: (i) impact of simple behavior modification on infant mortality, (ii) critical time window for susceptibility, (iii) low overall incidence in the population, and (iv) limited access to postmortem tissues. These factors, along with differences in gender, ethnicity, and infection, present significant challenges for identifying statistically significant molecular differences that may impact survival. Hence, to better understand the wealth of existing data and focus research efforts, we require a comprehensive biological model of SIDS that accounts for candidate factors, phenotypes, and interactions. Such models will be critical for prioritizing the genes, interactions, and pathways that could lead us to the underlying genetic determinants of SIDS.

Because disease research and discovery rapidly accelerate, it is essential to maintain a model that can be augmented and corrected over time. WikiPathways is an online repository of community-curated biological pathways that includes online and offline tools for editing and annotation. As opposed to gene lists or ontology categories, a WikiPathway model includes qualitative interactions and annotations that can be more intuitively understood by the typical laboratory biologist. Unlike static-pathway images, such as those presented in a typical review article, a WikiPathway contains structured relationships and embedded gene, metabolite, and reference information that can be updated by any researcher. Any WikiPathway can be reverted to a previous version or compared between distinct versions using built-in visualization tools. Because a WikiPathway can be exported to multiple formats and read by pathway- and network-analysis programs such as GenMAPP (15), PathVisio (16), and Cytoscape (17), it can be merged 


\section{Systems-level perspective of SIDS $\quad$ Integrated Mechanism Review}
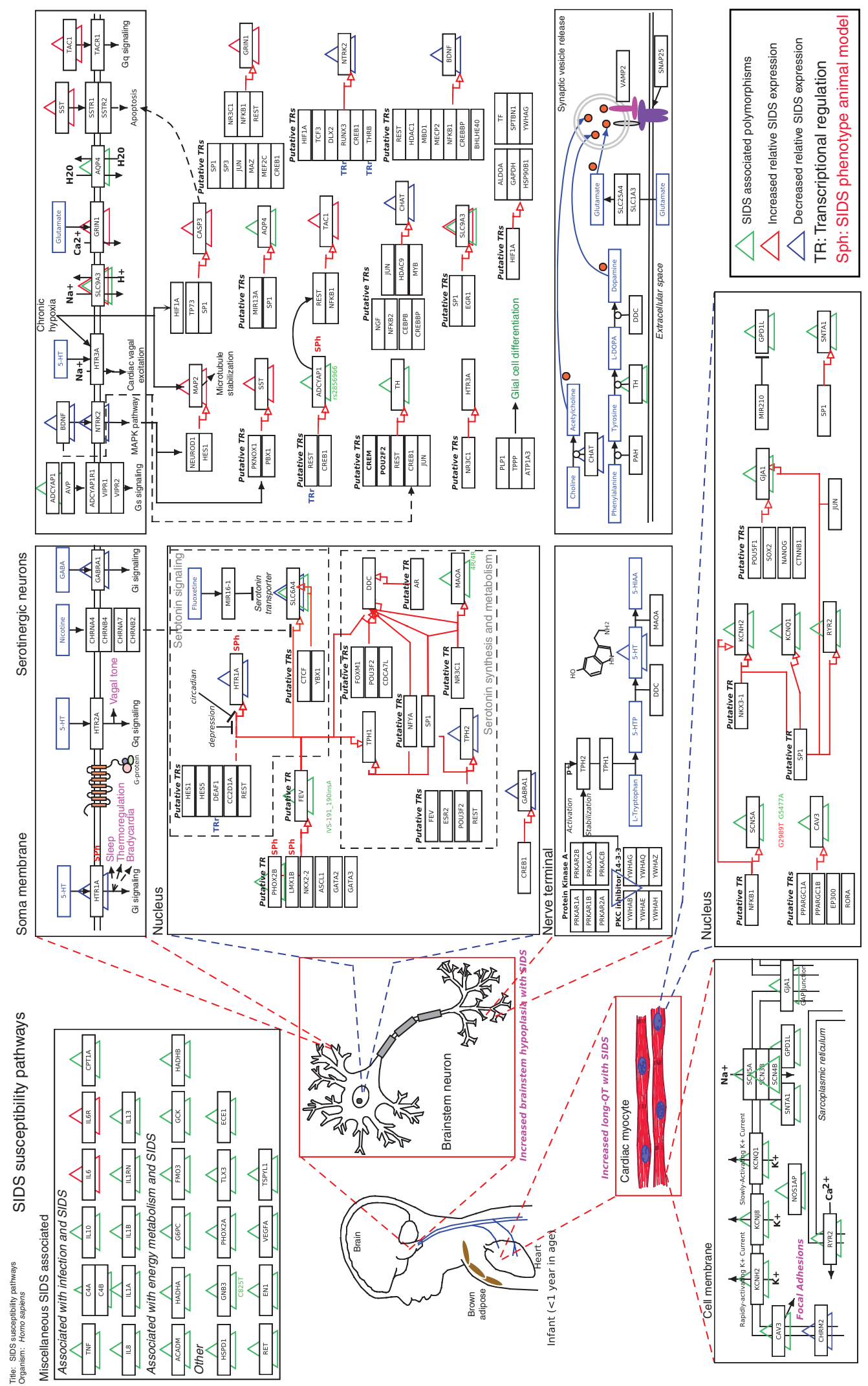

Figure 1. Integrated sudden infant death syndrome (SIDS) susceptibility pathway. Graphic export of the WikiPathway model of SIDS susceptibility (http://www.wikipathways.org/index.php/Pathway:WP706 revision 70114). A comprehensive list of DOI citations, associated gene identifiers, interactions, and metabolites are present in the online version (76). Red arrows denote transcriptional regulation. Genes are indicated by black nodes and other molecules by blue. Experimentally defined SIDS-associated molecules are indicated by an overlapping triangle denoting the type of evidence (genetic $=$ green increased abundance $=$ red, decreased abundance $=$ blue). Reprinted through Creative Commons Attribution 3.0 Unported license. Available at http://creativecommons.org/licenses/by/3.0. 
with quantitative whole-genome expression, epigenetic, polymorphism, protein or metabolic data, thus allowing for highly integrated and advanced data interpretation.

In this study, we developed an integrated view of SIDS as a WikiPathway (Figure 1). The purpose of this model is threefold: (i) to serve as an overview of the research in this field, (ii) to identify novel crosstalk between implicated molecular components, and (iii) to recommend new candidates for focused experimental analyses. For genome-wide analyses, such candidates would further allow for prioritization of regulated genes that directly or indirectly interact with molecular components relevant to SIDS biology. Through computational analysis of transcriptional targets, we identified previously implicated and novel upstream regulators of SIDS-associated factors. Integration of this pathway model with a recently published proteomics analysis of SIDS and control brainstem samples allowed us to identify novel biological markers linked to known SIDS pathology that may eventually provide novel mechanistic insights into SIDS susceptibility.

\section{METHODS}

\section{SIDS WikiPathway Creation}

A comprehensive survey of the literature was performed from the PubMed database using the search terms "Sudden Infant Death", along with "protein", "gene", "RNA", "DNA", "variant", or "genetic", yielding 249 associated genes from 1,141 articles. Results from all species were considered. After a careful examination of the literature for each gene, only 67 were considered to have sufficient evidence for involvement in SIDS based on the reported conclusions of the manuscript authors and supplied statistical evidence (see Supplementary File S1 online). Associated genes, proteins, and metabolites were further segregated based on associated cell-type or organ system, i.e., neuronal, cardiac, immune, or other system.

Additional biological interactions were included based on the literature (protein or DNA interactions) or using the computational tool GO-Elite to identify common candidate upstream transcriptional regulators [PAZAR and Amadeus databases (18-20)]. Genes were then further organized based on cellular compartment (e.g., membrane, cytoplasm, postsynaptic terminal, and nucleus). All relevant literature references were included.

\section{Proteomics Analysis}

Raw proteomic data were generously provided by Hannah Kinney (Harvard University) in the form of an MS-Excel sheet. This dataset is also available as supplemental information in the work by Broadbelt et al. (21). In this study, brain samples from the gigantocellularis nucleus of the medial reticular formation of six confirmed infants with SIDS and four control ("acute") infants were isolated by micropunch. Infants with SIDS were specifically selected that possessed previously identified decreases in both TPH2 protein and 5-HT levels. Acute controls were defined as infants who died within $48 \mathrm{~h}$ of illness but not as a result of SIDS. A significant difference in the postmortem interval existed between the SIDS cases and acute controls (SIDS, $19.0 \pm 1.3 \mathrm{~h}$; acute controls, $14.0 \pm 3.9 \mathrm{~h}$ ). Although the authors were able to eliminate postmortem interval as a driving factor in the differential expression of 14-3-3 family of protein kinase inhibitors using a larger validation cohort, such an analysis was not performed for the remaining proteomics predictions evaluated in this small cohort. Hence, postmortem interval should be considered a potential confounding variable for these analyses. To compare and integrate these findings into our in silico SIDS model, we reanalyzed the published dataset of assigned protein spectral-count values output from the software Scaffold (version 3.1.2, Proteome Software, Portland, OR). One sample, SIDS:82, was excluded from this analysis because of the lower overall spectral counts relative to all other samples ( 878 counts vs. mean counts of 2,500 in the remaining set). Quantile normalization was applied to the remaining sample protein expression values (total spectral counts per protein) and all subsequent values incremented by 1 spectral count to obtain conservative fold changes. Gene annotations were obtained by translating the International Protein Index identifiers to Ensembl gene IDs, or where no Ensembl translation existed (in the Ensembl 62 and 65 databases), from the provided gene symbol. Moderated $t$-test $P$ values (Limma equivalent) were calculated from these normalized protein-expression values in AltAnalyze (22) (see Supplementary File S2 online). In total, 63 proteins were obtained from this analysis with a $P<0.1$, the same filter used by Broadbelt et al.; however, the original list of targets identified was unavailable for comparison. Enriched pathways, ontology terms, and other gene sets were identified for differentially regulated proteins using the GO-Elite analysis module of software AltAnalyze version 2.0.8.

\section{SIDS Pathway Extension}

A database of gene interactions and gene-to-pathway associations were obtained from curated biological pathways (WikiPathways, KEGG). Databases were downloaded on 8/1/2012. All annotated WikiPathways $(5,621)$ and KEGG-pathway $(20,259)$ interactions were obtained from PathVisio-formatted GPML files distributed at http:// www.wikipathways.org and at http://www.pathvisio.org, respectively. Novel interactions between the SIDS WikiPathway (core) and the SIDS regulated protein dataset (regulated) were obtained by identifying nodes 1-2 degrees away from the core and regulated nodes, based on the combined interaction database. Associations between core and regulated nodes were inferred by identifying the pathways shared by genes in these two groups. Statistical enrichment for the number of associated interactions obtained between the 63 regulated proteins and the 67 SIDS core markers was determined by comparing the observed number to that obtained for 10,0000 permuted randomized gene sets of the same size regulated set $(n=63)$.

\section{RESULTS}

\section{WikiPathway Creation}

Pathway design. Given the heterogeneity of SIDS-associated polymorphisms, animal genetics, and postmortem findings, our model focuses separately on relevant neurological, cardiac, and immunological interactions. For each of these cell/organ systems, genes and genotypes with the strongest reported SIDS associations were compiled from the literature and annotated in the pathway. Annotated protein-protein, protein-metabolite, microRNA-mRNA (i.e., translational repression) and protein-DNA (i.e., transcriptional regulation) interactions were extended out for each of the SIDS-associated genes. In total, 67 implicated SIDS factors and 57 interacting genes were assembled into the resulting WikiPathway (revision 49768). Although the majority of these observations were not re-evaluated in independent studies, these are included for the purpose of identifying broader candidate interactions. Custom graphics were used for each implicated cell or organ system as a means of intuitively organizing data from these different systems (Figure 1). Biological pathway-level enrichment analysis indicated that a large subset of these genes are involved in response to hypoxia, neuron projection, nervous system development, spinal cord injury, apoptosis, and localization to mitochondrion among others (see Supplementary File S1 online). Examination of disease ontologies indicated an enrichment in basal ganglia diseases, depressive/anxiety disorder, respiratory signs and symptoms, tauopathies, hypertension, and cerebrovascular disorders among others. 


\section{Systems-level perspective of SIDS}

SIDS and the central nervous system. Several lines of evidence indicate that SIDS has a strong autonomic nervous system component, in particular within neurons of the brainstem (23). The raphe nuclei of the medulla oblongata are strongly implicated in SIDS, where ultrastructural, cellular, and molecular changes have been reported in infants with SIDS (24). The raphe nuclei synthesize a large proportion of the serotonin that is produced in the human body and function in the regulation of normal cardiopulmonary activity (25), sleep (26), and thermoregulation (27).

In the proposed model, genes controlling serotonin synthesis and signal transduction were observed to have the strongest genetic and protein-expression associations with SIDS. Principal among these genes were the serotonin biosynthetic enzyme $\mathrm{TPH} 2$, the serotonin transporter SLC6A4, and the serotonin receptor HTR1A $(3,4,24,28)$. Using the software GO-Elite, we discovered that several of these genes were regulated by a common transcription factor, FEV (the human orthologue of PET1(5)). In mice, PET-1 knockout results in the loss of up to $90 \%$ of serotonin neurons (29), whereas polymorphisms in FEV are over-represented in African-American infants with SIDS (5).

Vasopressin signaling molecules were also included in our model as putative regulators of cardiopulmonary regulation (30). A protein that is associated with vasopressin signaling, named pituitary adenylate cyclase-activating polypeptide (gene name ADCYAP1), results in a SIDS-like phenotypecharacterized by a high increase in spontaneous neonatal death, exacerbated by hypothermia and hypoxia-when disrupted in mice (31). Pituitary adenylate cyclase-activating polypeptide is widely expressed throughout the central nervous system, including autonomic control centers (32), and seems to be polymorphic in African-American SIDS cases (6). Although it is not clear whether ADCYAP1 and HTR1A are expressed in the same cells, these proteins regulate opposing $G$ protein-coupled receptor signaling pathways.

Nicotinic acetylcholine receptors, which are expressed in serotoninergic raphe neurons throughout development (33), were also included in this model because of the potential implication in SIDS. The application of nicotine or exposure to cigarette smoke is sufficient to inhibit electrical activity of raphe serotoninergic neurons (34), and chronic nicotine infusion in rats decreases expression of the SLC6A4 gene (35). Furthermore, nicotine exposure reduces immunoreactivity of the serotonin receptors 5-HT1A (HTR1A) and 5-HT2A (HTR2A) in several nuclei of the brainstem (36).

Many neuronal-implicated SIDS genes (ADCYAP1 (37), HTR1A (38), TPH2 (39), GRIN1 (40), CHRNB2 (40), TH (41), and TAC1 (42)) can all be transcriptionally regulated by REST (repressor element-1 silencing transcription factor). REST, along with three other genes (HNF4A, SP1, and CREB1), was independently enriched in our transcriptional regulatory analysis, suggesting that these factors may be novel targets of interest in SIDS susceptibility.

SIDS and the heart. Several studies have identified a critical link between cardiac arrhythmia (i.e., long-QT syndrome) and

\section{Integrated Mechanism Review}

SIDS (9). Indeed, several research groups hypothesize that a subset of diagnosed SIDS deaths are identical in etiology to adult forms of cardiac sudden death (43). A number of genetic association studies identified function-modifying mutations in critical cardiac channels in as many as $15 \%$ of all SIDS cases (9). These mutations have been predicted to predispose infants to lethal cardiac arrhythmias and cardiomyopathies associated with sudden death. The highest proportion of SIDS-associated mutations (both inherited and sporadic) is found in the sodium channel gene SCN5A. SCN5A mutations cause longQT3 syndrome, which is responsible for the highest incidence of spontaneous death during sleep; therefore, this disorder may account for a significant proportion of SIDS deaths (44). Mutations in other crucial cardiac-signaling genes, including connexin-43 (GJA1), ryanodine receptor 2 (RYR), and several others, have also been implicated (9). In addition, further examination highlighted a diverse set of putative transcriptional regulators for these genes, as well as the relatively common transcription factor, SP1 (45-47).

SIDS and the immune system. Infection is considered a significant risk factor for SIDS (48), and several mutations have been identified in genes associated with inflammatory responses and thermoregulation. For mutations in inflammation-associated genes, such as TNF-alpha (10), interleukin (IL-10 (11)), and complement component-4 (12), many are only significant in the presence of infection and SIDS. In SIDS cases, cerebrospinal fluid levels of IL- 6 are also increased as are IL-6 receptor levels in the arcuate nucleus of the brain, another major site of serotonin synthesis (49). Genes such as IL-6 and ADCYAP1 have also been linked with inflammatory processes $(12,50)$, suggesting that pathways associated with inflammation in the brain can promote or predispose SIDS (49).

Other implicated SIDS genes. Regulation of thermogenesis by brown adipose tissue has been proposed to be an important component of SIDS, given that SIDS incidence is highest in the winter months and that animal models of SIDS demonstrate variations in body temperature compared with controls (51-53). Interestingly, the activation of raphe HTR1A decreases both shivering and peripheral vasoconstriction in piglets (27). Other miscellaneous genes with genetic associations for SIDS cases are also shown.

Replication of genetic findings. Although many of the indicated genes in our molecular model were implicated from small cohort analyses and often for rare variants, a number of these genetic observations have been re-evaluated in larger independent cohorts. Chief among these are the neuronal signaling components MAOA, PHOX2B, and SLC6A4, where multiple studies have evaluated specific variants in SIDS (54-62). Only in the case of MAOA are there independent studies, which demonstrate the significant enrichment of a genetic variant in SIDS cases (promoter length polymorphisms) relative to controls $(54,55)$; however, there are an equal number of studies that dispute this claim $(56,57)$. MAOA is an attractive 


\section{Integrated Mechanism Review}

candidate molecular regulator because it is encoded on the $\mathrm{X}$-chromosome, and hence, may influence the greater propensity of male infants to succumb to SIDS. Many other variants have been evaluated in replication studies; however, nearly all of these do not reach statistical significance on post hoc analyses or are borderline significant $(58,62-64)$. In the case of both IL-1 (65) and IL-1 receptor antagonist (66) gene variants, significant replication in a single study has been reported in SIDS.

An intriguing strategy to evaluate the penetrance of reported SIDS polymorphisms is in reference to the large and growing DNA sequence and single-nucleotide polymorphism databases. To evaluate the incidence of channelopathy-associated genetic variants for CAV3, $\mathrm{KCNH} 2$, and SCN5A in the general population, Andreasen et al. (67) recently evaluated these variants from Exome Sequencing Project analyzed subjects. The incidence of these variants was found to be high (1 in 29) relative to the known incidence of SIDS. However, these analyses do not consider the distribution of these variants in SIDS cases or the presence of extrinsic risk factors, such as sleep position, which are considered necessary for triggering SIDS in a large subset of cases.

\section{Integration With SIDS Proteomics Data}

All of the molecular associations discussed thus far were derived using a classical candidate gene or molecular approach. However, to identify novel regulators that could open new avenues for the mechanistic evaluation of SIDS, we require unbiased discovery using high-throughput experimental strategies such as genomics and proteomics. Recently, Broadbelt et al. (21) performed a proteomic survey of the brainstem of a small cohort of infants who died of SIDS. The infants with SIDS evaluated in this study all had prior detected decreases in the amount of TPH2 and 5-HT. Although their analysis focused on alterations in the 14-3-3 family of protein kinase inhibitors occurring in SIDS compared with control infants, several dozen additional proteins were predicted to be regulated (list unpublished).

Although only a small cohort of samples were analyzed by Broadbelt et al. using proteomics, we reanalyzed this data to determine whether any regulated peptides can be bioinformatically implicated in known SIDS pathways or with our presented integrated model. Such an analysis further provides an important workflow for augmenting the presented WikiPathway model. Similar to the study by Broadbelt et al., we identified 63 proteins that achieved a $P<0.1$ moderated $t$-test cutoff when comparing SIDS vs. acute controls. Similar to the original analysis, 14-3-3 protein family members were among the most significant proteins identified (see Supplementary File S2 online). To discover functionally informative gene sets, we performed GO-Elite analysis on upregulated and downregulated genes for pathways, ontologies, transcription targets, and cellular biomarkers. This analysis revealed a number of gene sets associated with SIDS pathology, including those that regulate synaptic transmission (SNAP25, VAMP2, SLC1A3, and SLC25A4), myelin sheath development and glial cell differentiation (GAP43, MBP, PLP1, TPPP, ATP1A3, and TUBB4A),

\section{Salomonis}

and response to hypoxia (ALDOA, GAPDH, HSP90B1, TF, SPTBN1, and YWHAG). Novel categories discovered include gene sets involved in glycolysis and gluconeogenesis (ALDOA, GAPDH, GOT1, and GOT2), the tricarboxylic acid cycle (ACO2 and IDH2), prolactin signaling (PPIA, SIRPA, YWHAG, and YWHAZ), cytoskeletal organization (KRT17, KRT2, KRT9, MAPT, MSN, SPTBN1, and TUBB), and transcriptional regulation by the transcription factors NFIA (ALDOA, MBP, and PLP1), SP1 (ALDOA, ATP1A3, and MBP), HSF1 (ATP1B1, CRYM, and HSPA1A), FOS (GOT1, MBP, STIP1, and TUBB4B) and HNF4A (ANXA6, GAPDH, GNB1, GOT1, HSP90B1, and TF) (see Supplementary File S1 online).

Synaptic transmission-associated proteins collectively had the largest observed fold changes in this dataset. SNAP25 and VAMP2 protein expression was downregulated 7- and 3.5-fold, respectively, in SIDS vs. acute controls $\left({ }^{* *} P<0.01\right)$. In addition, the neurotransmitter reuptake transporters SLC25A4 and SLC1A3 were downregulated 4.8- and 3.9-fold, respectively $\left({ }^{\star} P<0.05\right)$ (Figure 2a).

\section{Connecting Proteomics Results With Validated SIDS Markers}

To evaluate how proteins identified through the proteomics approach can interact with more established SIDS markers, we identified genes in both sets that participate in the same biological pathways or share annotated interactions. All known SIDS markers were considered for these two queries.

For the analysis of shared pathways, 29 of 67 established SIDS markers were found to overlap with 30 of the 63 targets identified from the brainstem proteomics analysis in one or more pathways (23 common pathways in all) (Table 1). An example is the co-occurrence of MBP (myelin basic protein) and the transcription factor PHOX2B in the neural crest differentiation pathway. Both MBP and PHOX2B are regulated by the transcription factor SOX10 in this pathway. This pathway co-occurrence analysis identified several additional factors that would not have been connected to SIDS biology using other methodologies.

When possible annotated interactions between established SIDS markers and proteomics findings were examined, four genes (VAMP2, RAP1B, MAPT, and MBP) were found to connect indirectly to the gap junction protein connexin-43 (GJA1) through protein kinase or MAP kinase intermediates (Figure 3C). In addition, the genes MAPT and SLC directly and indirectly interact through the SIDS associated protein CASP3, respectively. These interactions were statistically enriched when compared with a permuted set of random proteins relative to SIDS annotated markers $(P=0.04)$.

\section{DISCUSSION}

Although the incidence of SIDS has decreased substantially through educational campaigns that promote safe sleep, the number of SIDS deaths remains high. Improvements in educational efforts and legislation around safe sleep are still needed, as are diagnostic methods to identify at-risk infants in need of stringent monitoring or possible prophylactic intervention 
a

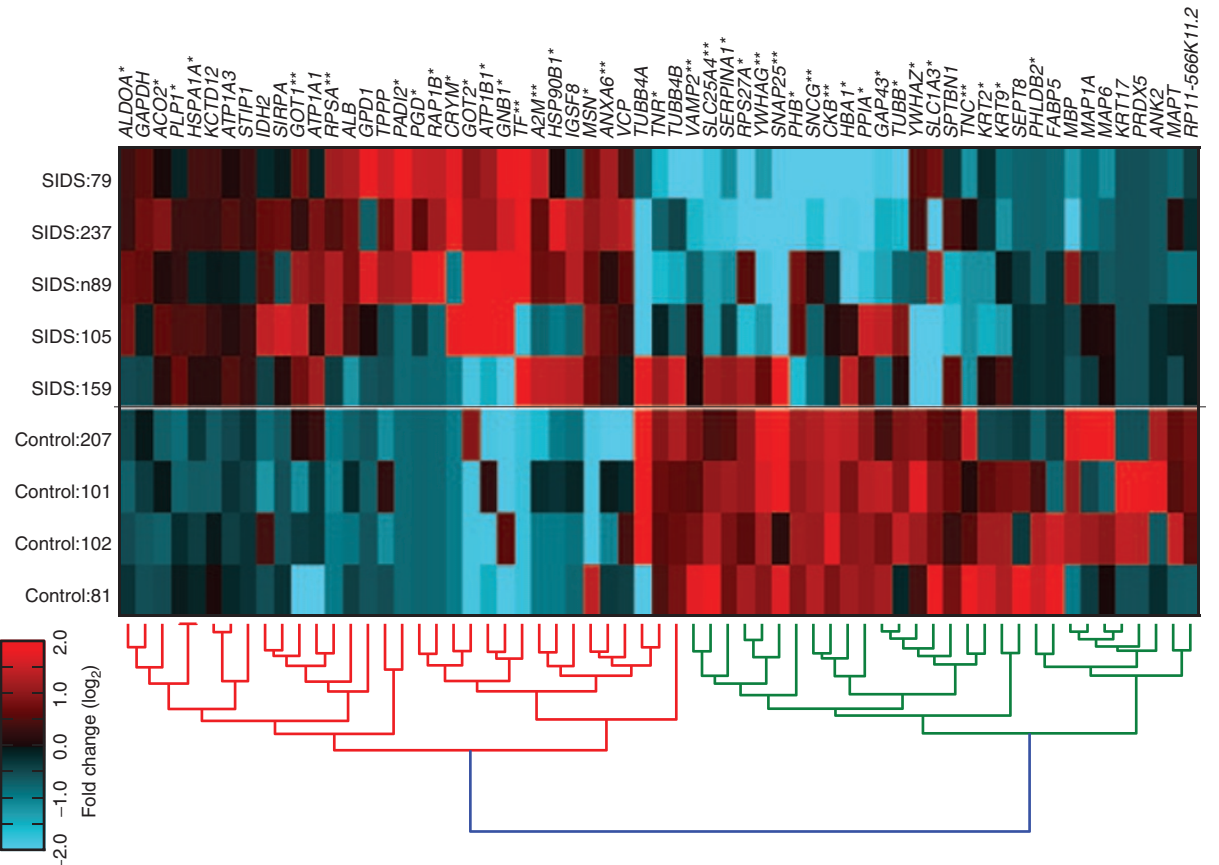

b
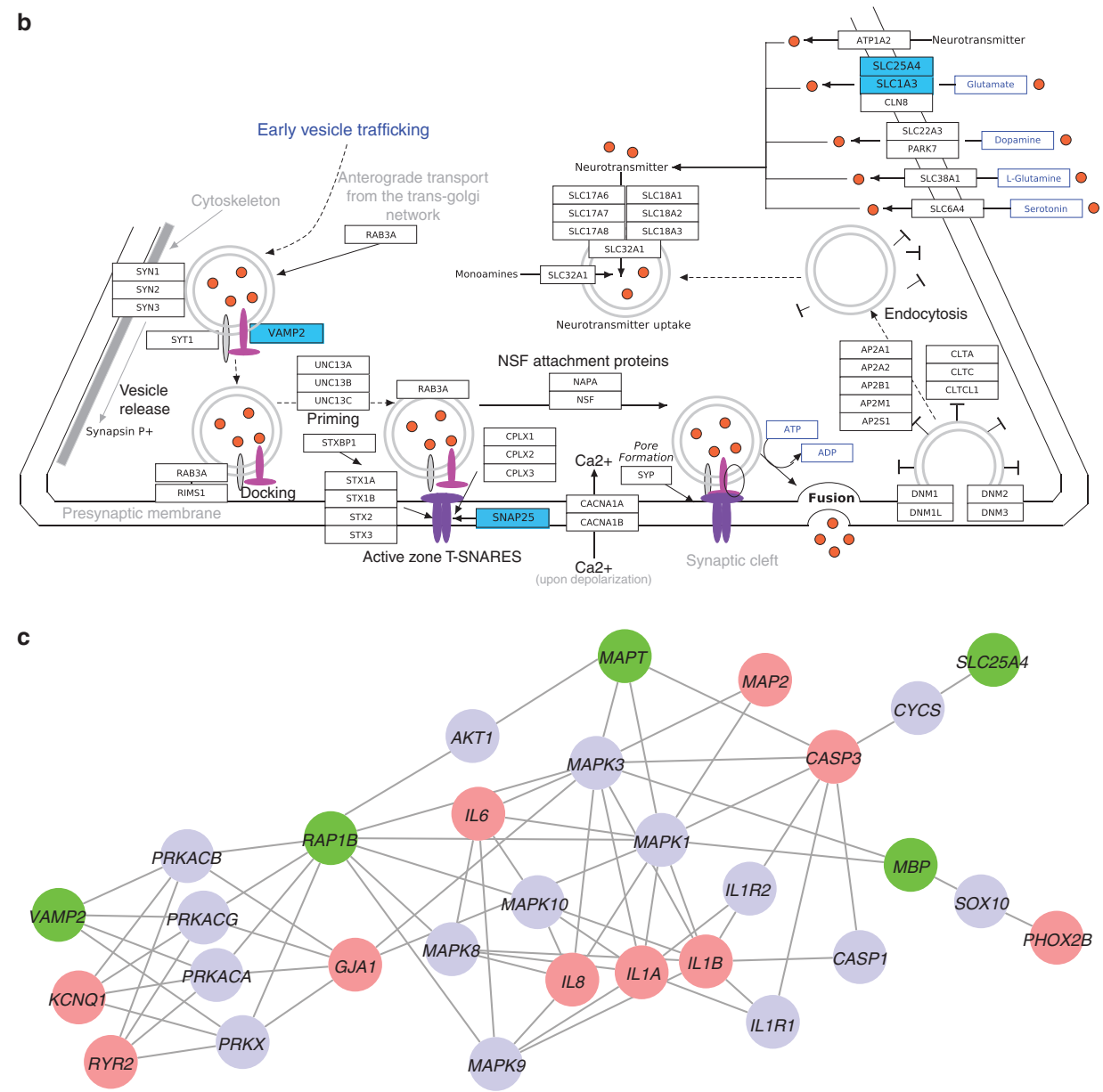

Figure 2. Novel proteomic sudden infant death syndrome (SIDS) associations. Differentially regulated proteins identified through re-analysis of SIDS brainstem proteomic profiles. (a) AltAnalyze produced hierarchically clustered SIDS and acute control protein-expression profiles relative to the mean of all samples; blue and red indicate up- and downregulation, respectively $\left({ }^{*} P<0.05,{ }^{* *} P<0.01\right)$. (b) AltAnalyze visualization of the WikiPathways Synaptic Vesicle Pathway (WP2267), with proteins downregulated in the SIDS proteomic analysis in blue. (c) Annotated interactions between differentially expressed proteins in SIDS cases (green), previously implicated SIDS genes (light red), and interacting proteins (light blue) in Cytoscape. 


\section{Integrated Mechanism Review | Salomonis}

Table 1. Implicated SIDS genetic loci and observed SIDS-regulated brainstem proteins occurring within common biological pathways (KEGG or WikiPathways)

\begin{tabular}{|c|c|c|}
\hline Shared pathway & SIDS implicated & SIDS regulated proteins \\
\hline Protein digestion and absorption: KEGG-hsa04974 & KCNQ1, SLC9A3 & ATP1B1, ATP1A1, ATP1A3 \\
\hline Pancreatic secretion: KEGG-hsa04972 & KCNQ1, RYR2 & ATP1B1, RAP1B, ATP1A1, ATP1A3 \\
\hline PPAR signaling pathway: KEGG-hsa 03320 & ACADM, CPT1A & FABP5 \\
\hline Nicotine activity on dopaminergic neurons:WP1602 & CHRNA4, CHRNB2, TH & GNB1 \\
\hline Glutamatergic synapse: KEGG-hsa04724 & GNB3, GRIN1 & GNB1, SLC1A3 \\
\hline Malaria: KEGG-hsa05144 & IL10, IL1B, IL6, IL8, TNF & HBA1 \\
\hline Selenium pathway:WP15 & IL1B, IL6 & HBA1, PRDX5, ALB \\
\hline Pathways in cancer: KEGG-hsa05200 & CASP3, IL6, IL8, RET, VEGFA & HSP90B1 \\
\hline Endocytosis: KEGG-hsa04144 & CAV3, RET & HSPA1A \\
\hline Measles: KEGG-hsa05162 & IL1A, IL1B, IL13, IL6 & MSN, HSPA1A \\
\hline GPCRs, class A rhodopsin-like:WP455 & CHRM2, HTR1A, HTR2A & RP11-566K11.2 \\
\hline Calcium signaling pathway: KEGG-hsa04020 & CHRM2, CHRNA7, GRIN1, HTR2A, RYR2 & SLC25A4 \\
\hline Mineral absorption: KEGG-hsa04978 & SLC9A3 & TF, ATP1B1, ATP1A1, ATP1A3 \\
\hline Focal adhesion: KEGG-hsa04510 & CAV3, VEGFA & TNC, TNR, RAP1B \\
\hline Gap junction: KEGG-hsa04540 & GJA1, HTR2A & TUBB, TUBB4B, TUBB4A, RP11-566K11.2 \\
\hline Calcium regulation in the cardiac cell:WP536 & CHRM2, GJA1, GNB3, RYR2 & $\begin{array}{l}\text { YWHAG, ANXA6, ATP1B1, YWHAZ, } \\
\text { GNB1 }\end{array}$ \\
\hline Myometrial relaxation and contraction pathways:WP289 & GJA1, GNB3, IL1B, IL6, RYR2 & YWHAG, YWHAZ, GNB1 \\
\hline
\end{tabular}

GPCRs, G protein-coupled receptors; SIDS, sudden infant death syndrome.

(e.g., prenatal diagnosis of long-QT syndrome). Such personalized medicine approaches are likely to be required given the apparent genetic heterogeneity and penetrance of SIDS. As demonstrated by the analysis of colon cancer genotypes, pathway-based prioritization of disease variants is a potent strategy for developing new diagnostic models to understand how seemingly heterogeneous genetic elements can contribute to a common biological endpoint (68). There are several challenges in the development of a pathway model for SIDS, including the unclear role of genetic factors, multiple implicated organ systems, limited and week associations based on few cases, and investigator bias in gene evaluation.

The SIDS WikiPathway resource described herein represents an important starting point from which to begin evaluating next-generation genomic profiles in a focused way, to identify events that may not immediately seem to be related. As an example, we analyzed brainstem proteomics data from infants who died of SIDS or other causes. From these data, we identified a large number of genes that could be connected to previously implicated SIDS genetic loci based on known biological pathway interactions. More importantly, we identified a number of proteins that regulate key biological processes associated with SIDS pathophysiology, such as brainstem gliosis, hypoxia, and inhibition of synaptic vesicle fusion. We subsequently used both sets of results to improve our SIDS WikiPathway model. Finally, through the restricted analysis of established SIDS genetic loci, we discovered key regulatory factors and potential crosstalk mechanisms between distinct SIDS gene associations that would have been difficult without a pathway-based model. Among these, FEV, REST, HNF4A, and SP1 require additional attention as transcription factors that could drive control elements for established SIDS genes. SP1 and HNF4A were enriched in both literature-associated SIDS genes and among SIDS brainstem differentially expressed proteins.

Potential crosstalk between the serotonin and vasopressin pathways will also require additional exploration. Several of the genes identified through our proteomics re-analysis directly impinge upon cell types associated with the gross phenotypic alterations observed in infants with SIDS (69). One of the most intriguing alterations we observed was in the regulation of factors associated with oligodendrocyte differentiation and myelin deposition. Three of these genes were upregulated in SIDS (PLP1, TPPP, and ATP1A3) and three were downregulated (GAP43, MBP, and TUBB4A). An early discovery into the pathology of SIDS was the finding that infants with SIDS have a significantly increased number of glial cells within the brainstem, which may act to disrupt neuronal growth $(69,70)$. GAP43 expression is known to be downregulated during 


\section{Systems-level perspective of SIDS}

oligodendrocyte differentiation and is likely to be a marker of earlier-stage progenitor cells (71). Differentiating oligodendrocytes are believed to be among the most metabolically active cell types in the brain, owing to the energy required for filopodial extension and myelin ensheathment of axons $(72,73)$. In line with this observation, we saw the coordinate and relatively large upregulation of factors promoting glycolysis and the tricarboxylic acid. We also observed the upregulation of the filopodia-associated gene moesin (MSN) in SIDS cases $\left({ }^{*} P<0.05\right)$. These factors represent interesting and possibly causative regulators of increased glial cell production the SIDS brainstem.

Another important observation from this proteomics dataset was the coordinate downregulation of factors involved in synaptic transmission, particularly the synaptic vesicle-associated proteins SNAP25 and VAMP2. Botulinum spores cleave both SNAP25 and VAMP2, and exposure to this toxin is responsible for a subset of documented SIDS cases (74). However, this downregulation could also happen through a regulatory mechanism independent of external pathogens. In addition, the increased expression of a number of HIF1-alpha targets suggests that chronic hypoxia may be a contributing pathology in the SIDS brains analyzed in this study. HIF1-alpha expression is induced during hypoxia, to increase the expression of target genes believed to promote oxygen delivery or facilitate adaptation to hypoxic conditions. Chronic hypoxia is believed to be a common trigger of SIDS (75).

It is important to note that the genes observed in these proteomics analyses have insufficient statistical evidence to definitely associate these with SIDS, because of the small number of samples analyses, relatively small number of peptides detected, and the potential effect of differing postmortem interval. The same is true for the majority of genetic studies reported in this review, which are typically not validated on an independent cohort of sufficient size to definitely associate a given polymorphism with SIDS. Hence, it is critical for researchers to be able to evaluate existing findings within a coherent framework as well as identify in silico predicted regulators for focused validation. We believe the SIDS pathway model described in this review will provide an important reference for such future studies and allow for additional connectivity analyses to novel factors. Such studies will inevitability improve this community in silico model as a result.

\section{SUPPLEMENTARY MATERIAL}

Supplementary material is linked to the online version of the paper at http://www.nature.com/pr

\section{ACKNOWLEDGMENT}

We thank Anna Lisa Lucido at the Gladstone Institutes for her editorial assistance and helpful discussions relating to the data analysis.

\section{STATEMENT OF FINANCIAL SUPPORT}

This work was supported by the National Institutes of Health (Bethesda, MD) grant GM080223-06S1.

Disclosure: The author has no financial ties to products reviewed in this study or potential/perceived conflicts of interest.

\section{Integrated Mechanism Review}

\section{REFERENCES}

1. Task Force on Infant Sleep Position and Sudden Infant Death Syndrome. Changing concepts of sudden infant death syndrome: implications for infant sleeping environment and sleep position. American Academy of Pediatrics. Pediatrics 2000;105:650-6.

2. Kinney HC, Richerson GB, Dymecki SM, Darnall RA, Nattie EE. The brainstem and serotonin in the sudden infant death syndrome. Annu Rev Pathol 2009;4:517-50.

3. Narita N, Narita M, Takashima S, Nakayama M, Nagai T, Okado N. Serotonin transporter gene variation is a risk factor for sudden infant death syndrome in the Japanese population. Pediatrics 2001;107:690-2.

4. Weese-Mayer DE, Berry-Kravis EM, Maher BS, Silvestri JM, Curran ME, Marazita ML. Sudden infant death syndrome: association with a promoter polymorphism of the serotonin transporter gene. Am J Med Genet A 2003;117A:268-74.

5. Hendricks T, Francis N, Fyodorov D, Deneris ES. The ETS domain factor Pet-1 is an early and precise marker of central serotonin neurons and interacts with a conserved element in serotonergic genes. J Neurosci 1999;19:10348-56.

6. Cummings KJ, Klotz C, Liu WQ, et al. Sudden infant death syndrome (SIDS) in African Americans: polymorphisms in the gene encoding the stress peptide pituitary adenylate cyclase-activating polypeptide (PACAP). Acta Paediatr 2009;98:482-9.

7. Filonzi L, Magnani C, Lavezzi AM, et al. Association of dopamine transporter and monoamine oxidase molecular polymorphisms with sudden infant death syndrome and stillbirth: new insights into the serotonin hypothesis. Neurogenetics 2009;10:65-72.

8. Weese-Mayer DE, Berry-Kravis EM, Zhou L, et al. Sudden infant death syndrome: case-control frequency differences at genes pertinent to early autonomic nervous system embryologic development. Pediatr Res 2004;56:391-5.

9. Tester DJ, Ackerman M. Cardiomyopathic and channelopathic causes of sudden, unexpected death in infants and children. Annu Rev Med 2009;60:69-84.

10. Ferrante L, Opdal SH, Vege A, Rognum TO. TNF-alpha promoter polymorphisms in sudden infant death. Hum Immunol 2008;69:368-73.

11. Perskvist N, Skoglund K, Edston E, Bäckström G, Lodestad I, Palm U. TNF-alpha and IL-10 gene polymorphisms versus cardioimmunological responses in sudden infant death. Fetal Pediatr Pathol 2008;27:149-65.

12. Opdal SH, Vege A, Stave AK, Rognum TO. The complement component C4 in sudden infant death. Eur J Pediatr 1999;158:210-2.

13. Rahim RA, Boyd PA, Ainslie Patrick WJ, Burdon RH. Human heat shock protein gene polymorphisms and sudden infant death syndrome. Arch Dis Child 1996;75:451-2.

14. Yang Z, Lantz PE, Ibdah JA. Post-mortem analysis for two prevalent betaoxidation mutations in sudden infant death. Pediatr Int 2007;49:883-7.

15. Salomonis N, Hanspers K, Zambon AC, et al. GenMAPP 2: new features and resources for pathway analysis. BMC Bioinformatics 2007;8:217.

16. van Iersel MP, Kelder T, Pico AR, et al. Presenting and exploring biological pathways with PathVisio. BMC Bioinformatics 2008;9:399.

17. Cline MS, Smoot M, Cerami E, et al. Integration of biological networks and gene expression data using Cytoscape. Nat Protoc 2007;2:2366-82.

18. Zambon AC, Gaj S, Ho I, et al. GO-Elite: a flexible solution for pathway and ontology over-representation. Bioinformatics 2012;28:2209-10.

19. Linhart C, Halperin Y, Shamir R. Transcription factor and microRNA motif discovery: the Amadeus platform and a compendium of metazoan target sets. Genome Res 2008;18:1180-9.

20. Portales-Casamar E, Kirov S, Lim J, et al. PAZAR: a framework for collection and dissemination of cis-regulatory sequence annotation. Genome Biol 2007;8:R207.

21. Broadbelt KG, Rivera KD, Paterson DS, et al. Brainstem deficiency of the 14-3-3 regulator of serotonin synthesis: a proteomics analysis in the sudden infant death syndrome. Mol Cell Proteomics 2012;11:M111.009530.

22. Emig D, Salomonis N, Baumbach J, Lengauer T, Conklin BR, Albrecht M. AltAnalyze and DomainGraph: analyzing and visualizing exon expression data. Nucleic Acids Res 2010;38:W755-62. 
23. Ozawa Y, Takashima S. Developmental neurotransmitter pathology in the brainstem of sudden infant death syndrome: a review and sleep position. Forensic Sci Int 2002;130:Suppl:S53-9.

24. Lavezzi AM, Casale V, Oneda R, Weese-Mayer DE, Matturri L. Sudden infant death syndrome and sudden intrauterine unexplained death: correlation between hypoplasia of raphé nuclei and serotonin transporter gene promoter polymorphism. Pediatr Res 2009;66:22-7.

25. Messier ML, Li A, Nattie EE. Inhibition of medullary raphe serotonergic neurons has age-dependent effects on the $\mathrm{CO} 2$ response in newborn piglets. J Appl Physiol (1985) 2004;96:1909-19.

26. Messier ML, Li A, Nattie EE. Muscimol inhibition of medullary raphé neurons decreases the $\mathrm{CO} 2$ response and alters sleep in newborn piglets. Respir Physiol Neurobiol 2002;133:197-214.

27. Brown JW, Sirlin EA, Benoit AM, Hoffman JM, Darnall RA. Activation of 5-HT1A receptors in medullary raphé disrupts sleep and decreases shivering during cooling in the conscious piglet. Am J Physiol Regul Integr Comp Physiol 2008;294:R884-94.

28. Duncan JR, Paterson DS, Hoffman JM, et al. Brainstem serotonergic deficiency in sudden infant death syndrome. JAMA 2010;303:430-7.

29. Hendricks TJ, Fyodorov DV, Wegman LJ, et al. Pet-1 ETS gene plays a critical role in 5-HT neuron development and is required for normal anxietylike and aggressive behavior. Neuron 2003;37:233-47.

30. Pérgola PE, Alper RH. Vasopressin and autonomic mechanisms mediate cardiovascular actions of central serotonin. Am J Physiol 1991;260(6 Pt 2):R1188-93

31. Cummings KJ, Pendlebury JD, Sherwood NM, Wilson RJ. Sudden neonatal death in PACAP-deficient mice is associated with reduced respiratory chemoresponse and susceptibility to apnoea. J Physiol 2004;555(Pt 1):15-26.

32. Hannibal J. Pituitary adenylate cyclase-activating peptide in the rat central nervous system: an immunohistochemical and in situ hybridization study. J Comp Neurol 2002;453:389-417.

33. Duncan JR, Paterson DS, Kinney HC. The development of nicotinic receptors in the human medulla oblongata: inter-relationship with the serotonergic system. Auton Neurosci 2008;144:61-75.

34. Touiki K, Rat P, Molimard R, Chait A, de Beaurepaire R. Effects of tobacco and cigarette smoke extracts on serotonergic raphe neurons in the rat. Neuroreport 2007;18:925-9.

35. Semba J, Wakuta M. Chronic effect of nicotine on serotonin transporter mRNA in the raphe nucleus of rats: reversal by co-administration of bupropion. Psychiatry Clin Neurosci 2008;62:435-41.

36. Say M, Machaalani R, Waters KA. Changes in serotoninergic receptors $1 \mathrm{~A}$ and $2 \mathrm{~A}$ in the piglet brainstem after intermittent hypercapnic hypoxia (IHH) and nicotine. Brain Res 2007;1152:17-26.

37. Miyata A, Sugawara H, Iwata S, Shimizu T, Kangawa K. [The regulatory mechanism for neuron specific expression of PACAP gene]. Nihon Yakurigaku Zasshi 2004;123:235-42.

38. Le François B, Czesak M, Steubl D, Albert PR. Transcriptional regulation at a HTR1A polymorphism associated with mental illness. Neuropharmacology 2008;55:977-85.

39. Patel PD, Bochar DA, Turner DL, Meng F, Mueller HM, Pontrello CG. Regulation of tryptophan hydroxylase-2 gene expression by a bipartite RE-1 silencer of transcription/neuron restrictive silencing factor (REST/NRSF) binding motif. J Biol Chem 2007;282:26717-24.

40. Noh KM, Hwang JY, Follenzi A, et al. Repressor element-1 silencing transcription factor (REST)-dependent epigenetic remodeling is critical to ischemia-induced neuronal death. Proc Natl Acad Sci USA 2012;109:E962-71.

41. Kim SM, Yang JW, Park MJ, et al. Regulation of human tyrosine hydroxylase gene by neuron-restrictive silencer factor. Biochem Biophys Res Commun 2006;346:426-35.

42. Greco SJ, Smirnov SV, Murthy RG, Rameshwar P. Synergy between the RE-1 silencer of transcription and NFkappaB in the repression of the neurotransmitter gene TAC1 in human mesenchymal stem cells. J Biol Chem 2007;282:30039-50.

43. Tfelt-Hansen J, Winkel BG, Grunnet M, Jespersen T. Cardiac channelopathies and sudden infant death syndrome. Cardiology 2011;119:21-33.
44. Stramba-Badiale M, Priori SG, Napolitano C, et al. Gene-specific differences in the circadian variation of ventricular repolarization in the long QT syndrome: a key to sudden death during sleep? Ital Heart J 2000;1:323-8.

45. Lin $\mathrm{H}$, Xiao J, Luo X, et al. Overexpression HERG K(+) channel gene mediates cell-growth signals on activation of oncoproteins SP1 and NF-kappaB and inactivation of tumor suppressor Nkx3.1. J Cell Physiol 2007;212:13747.

46. Luo X, Xiao J, Lin H, Lu Y, Yang B, Wang Z. Genomic structure, transcriptional control, and tissue distribution of HERG1 and KCNQ1 genes. Am J Physiol Heart Circ Physiol 2008;294:H1371-80.

47. Nishida K, Otsu K, Hori M, Kuzuya T, Tada M. Cloning and characterization of the 5 -upstream regulatory region of the $\mathrm{Ca}(2+)$-release channel gene of cardiac sarcoplasmic reticulum. Eur J Biochem 1996;240:408-15.

48. Blood-Siegfried J, Bowers MT, Lorimer M. Is shock a key element in the pathology of sudden infant death syndrome (SIDS)? Biol Res Nurs 2009;11:187-94

49. Rognum IJ, Haynes RL, Vege A, Yang M, Rognum TO, Kinney HC. Interleukin- 6 and the serotonergic system of the medulla oblongata in the sudden infant death syndrome. Acta Neuropathol 2009;118:519-30.

50. Quintana A, Müller M, Frausto RF, et al. Site-specific production of IL-6 in the central nervous system retargets and enhances the inflammatory response in experimental autoimmune encephalomyelitis. J Immunol 2009;183:2079-88.

51. Audero E, Coppi E, Mlinar B, et al. Sporadic autonomic dysregulation and death associated with excessive serotonin autoinhibition. Science 2008;321:130-3.

52. Gray SL, Yamaguchi N, Vencová P, Sherwood NM. Temperature-sensitive phenotype in mice lacking pituitary adenylate cyclase-activating polypeptide. Endocrinology 2002;143:3946-54.

53. Li A, Nattie E. Serotonin transporter knockout mice have a reduced ventilatory response to hypercapnia (predominantly in males) but not to hypoxia. J Physiol 2008;586:2321-9.

54. Klintschar M, Heimbold C. Association between a functional polymorphism in the MAOA gene and sudden infant death syndrome. Pediatrics 2012;129:e756-61.

55. Courts C, Grabmüller M, Madea B. Monoamine oxidase A gene polymorphism and the pathogenesis of sudden infant death syndrome. J Pediatr 2013;163:89-93.

56. Opdal SH, Vege Å, Rognum TO. Genetic variation in the monoamine oxidase $A$ and serotonin transporter genes in sudden infant death syndrome. Acta Paediatr 2014;103:393-7.

57. Groß M, Bajanowski T, Vennemann M, Poetsch M. Sudden infant death syndrome (SIDS) and polymorphisms in Monoamine oxidase A gene (MAOA): a revisit. Int J Legal Med 2014;128:43-9.

58. Liebrechts-Akkerman G, Liu F, Lao O, et al. PHOX2B polyalanine repeat length is associated with sudden infant death syndrome and unclassified sudden infant death in the Dutch population. Int J Legal Med 2014;128:621-9.

59. Kijima K, Sasaki A, Niki T, et al. Sudden infant death syndrome is not associated with the mutation of PHOX2B gene, a major causative gene of congenital central hypoventilation syndrome. Tohoku J Exp Med 2004;203:658.

60. Paterson DS, Rivera KD, Broadbelt KG, et al. Lack of association of the serotonin transporter polymorphism with the sudden infant death syndrome in the San Diego Dataset. Pediatr Res 2010;68:409-13.

61. Haas C, Braun J, Bär W, Bartsch C. No association of serotonin transporter gene variation with sudden infant death syndrome (SIDS) in Caucasians. Leg Med (Tokyo) 2009;11:Suppl 1:S210-2.

62. Opdal SH, Vege A, Rognum TO. Serotonin transporter gene variation in sudden infant death syndrome. Acta Paediatr 2008;97:861-5.

63. Barrett KT, Rodikova E, Weese-Mayer DE, et al. Analysis of PAC1 receptor gene variants in Caucasian and African-American infants dying of sudden infant death syndrome. Acta Paediatr 2013; e-pub ahead of print 26 August 2013.

64. Opdal SH, Vege A, Stray-Pedersen A, Rognum TO. Aquaporin-4 gene variation and sudden infant death syndrome. Pediatr Res 2010;68:48-51. 
65. Ferrante L, Opdal SH, Vege A, Rognum TO. IL-1 gene cluster polymorphisms and sudden infant death syndrome. Hum Immunol 2010;71: 402-6.

66. Highet AR, Gibson CS, Goldwater PN. Variant interleukin 1 receptor antagonist gene alleles in sudden infant death syndrome. Arch Dis Child 2010;95:1009-12.

67. Andreasen C, Refsgaard L, Nielsen JB, et al. Mutations in genes encoding cardiac ion channels previously associated with sudden infant death syndrome (SIDS) are present with high frequency in new exome data. Can J Cardiol 2013;29:1104-9.

68. Network CGA. Comprehensive molecular characterization of human colon and rectal cancer. Nature 2012;487:330-7.

69. Solcher H. [Fibrillary glioses of brain stem in infancy? (author's transl)]. Acta Neuropathol 1973;26:81-4.

70. Kinney HC, Burger PC, Harrell FE Jr, Hudson RP Jr. 'Reactive gliosis' in the medulla oblongata of victims of the sudden infant death syndrome. Pediatrics 1983;72:181-7.
71. Curtis R, Hardy R, Reynolds R, Spruce BA, Wilkin GP. Down-regulation of GAP-43 during oligodendrocyte development and lack of expression by astrocytes in vivo: implications for macroglial differentiation. Eur J Neurosci 1991;3:876-86.

72. Jiang S, Seng S, Avraham HK, Fu Y, Avraham S. Process elongation of oligodendrocytes is promoted by the Kelch-related protein MRP2/KLHL1. J Biol Chem 2007;282:12319-29.

73. Lehotzky A, Lau P, Tokési N, Muja N, Hudson LD, Ovádi J. Tubulin polymerization-promoting protein (TPPP/p25) is critical for oligodendrocyte differentiation. Glia 2010;58:157-68.

74. Blasi J, Chapman ER, Link E, et al. Botulinum neurotoxin A selectively cleaves the synaptic protein SNAP-25. Nature 1993;365:160-3.

75. Bulterys MG, Greenland S, Kraus JF. Chronic fetal hypoxia and sudden infant death syndrome: interaction between maternal smoking and low hematocrit during pregnancy. Pediatrics 1990;86:535-40.

76. SIDS Susceptibility Pathways (Homo sapiens). http://www.wikipathways. org/index.php?title=Pathway:WP706\&oldid=70114. Accessed 30 July 2013. 